\title{
Composite reinforced metallic cylinder for high-speed rotation
}

\author{
S. Pradhan \\ Department of Chemical Engineering, Indian Institute of Science, Bangalore-560 012, India
}

The objective of the present study is to design and development of the composite reinforced thin metallic cylinder to increase the peripheral speed significantly and thereby improve the separation performance in a centrifugal gas separation processes through proper optimization of the internal parameters. According to Dirac equation (Cohen (1951)), the maximum separative work for a centrifugal gas separation process increase with $4^{\text {th }}$ power of the peripheral speed. Therefore, it has been intended to reinforce the metallic cylinder with composites (carbon fibers: T-700 and T- 1000 grade with suitable epoxy resin) to increase the stiffness and hoop stress so that the peripheral speed can be increased significantly, and thereby enhance the separative output.

Here, we have formulated the mathematical model to investigate the elastic stresses of a laminated cylinder subjected to mechanical, thermal and thermo-mechanical loading. A detailed analysis is carried out to underline the basic hypothesis of each formulation. Further, we evaluate the steady state creep response of the rotating cylinder and analyze the stresses and strain rates in the cylinder.

\section{Introduction}

Centrifugal gas separation processes effect separation by utilising the difference in the mole fraction in a high speed rotating cylinder caused by the difference in molecular mass, and consequently the centrifual force density. These have been widely used in isotope separation because chemical separation methods cannot be used to separate isotopes of the same chemical species. Radioactive isotopes, including zinc, cadmium, iridium, selenium, tellurium, germanium and molybdenum, are used in medical and industrial applications. In medical applications, radio-isotopes are used both for diagnostics such as gallium scans for locations of inflammation and infection, indium for leukocyte imaging and molybdenum/technetium scans for high bone growth, as well as for therapy including cancer therapy using isotopes of iodine and iridium. Nuclear diagnostic imaging has an important role in the identification and management of conditions such as heart disease, brain disorder, lung and kidney functions and a broad range of cancers. The high sensitivity and specificity of nuclear diagnostic imaging techniques offer the important advantages of being able to identify diseases at an early stage, to track disease progression, to allow for accurate disease staging and to provide predictive information about likely success of alternative therapy options. One of the important diagnostic techniques is gamma imaging. Gamma cameras are used to detect diseases of various organs including heart, brain, bone, lung and the thyroid using radio-isotope of gallium, indium, and iodine. Natural titanium is being used to encapsulate radioactive seeds for brachytherapy, in particular for prostate cancer. Depleted molybdenum is used in the experimental process of transmutation of the long-lived radio-isotopes, which are present in spent nuclear fuel. Cadmium isotope is employed as a precursor for the important radio pharmaceutical 
isotope indium, which has many varied medical applications. The cadmium isotope is placed in a cyclotron, and thereby is exposed to proton bombardment. The cadmium captures a proton then releases two neutrons such that indium is formed. Radioactive indium is used for several purposes such as a detector (of heart transplant rejection, of osteomyelitis and with gallium of soft tissue infection); imaging (of abdominal infections, of white blood cells, and of tumours), antibody labelling, cellular dosimetry, myocardial scans, and finally for the treatment of leukaemia. Cadmium isotopes are also being used in helium-cadmium lasers. Isotopically pure cadmium boost the output of the laser and also increase its coherence length. Iridium isotope is used in industrial applications for the non-destructive testing of constructions. Furthermore, iridium isotopes are being used for many medical applications such as brachytherapy for the treatment of prostate, brain, breast and gynaecological cancers. Xenon isotope is used as a precursor for radioactive iodine, which is an important thryroid diagnostic radiopharmaceutical isotope, and also used for diagnostic analysis of brain, kidney and neurological diseases. Iodine isotope is also used in diagnostic imaging and detection of osteoporosis, and in radiotherapy treatment of prostate cancer through seed implantation. An important industrial application is the separation of uranium isotopes for nuclear power generation. Other important application include the use of zinc isotopes to reduce corrosion in reactor cooling systems.

The objective of the present analysis is to develop the composite reinforced thin metallic cylinder which can rotate at very high peripheral speed (2 times higher than the metallic cylinder) for the improvement of separation performance in a centrifugal gas separation processes.

The continuing quest for improved performance, specified in terms of weight reduction, high strength and low cost has led to development of a new class of materials called composite materials. A composite material consists of combining two or more constituents called matrix and reinforcement. The constituents are combined at microscopic level and are not soluble to each other. Matrix phase materials are generally continuous while the reinforcing phase may be in the form of fibers, particles or flakes. The composite materials possess characteristic properties, such as high stiffness, high strength,low weight, high temperature performance, good corrosion resistance, high hardness and conductivity that are not possible in any of its constituents alone. Analysis of these properties reveals that they depend on the following parameters: (a)properties of the individual constituents, (b)relative amounts of the constituents, (c)size and shape of the constituents (i.e.Morphology), (d)degree of bonding between constituents, (e)orientation of the various constituents.

The composite materials possess high specific modulus and specific strength as compared to conventional materials. For selecting a composite material for a particula application, the following parameters to be considered: (i) strength, (ii) toughness, (iii) formadibility, (iv)weldeability, (v)corrosion resistance, and (vi) wear resistance.

The mechanical performance of a composite materials depends on number of factors like, (i) fibe grade, (ii) type of matrix, (iii) fibe matrix interface. Fibe grade involves (a) filament length, (b) filament diameter, (c) orientation, (d) shape, (e) tensile strength, (f) tensile modulus, (g) strain, (h) density, (i) yield strength, (j) specific heat, (k) thermal conductivity, (l) electric resistivity, and (m) chemical composition. The matrix functions include binding of the fibers together, protecting the fibers from environmental conditions, shielding the fibers from damage during handling and helps for transferring the load from matrix to the fiber. Apart from fibe and matrix there are several othe factors which affect the mechanical performance of composite materials. The fiber matrix interface is an important factor which determines how well the matrix transfers the loads 
to the fibers. The fibe matrix interfacial bonding is of following three types: a)Chemical Bonding, b)Mechanical Bonding, and c)Reaction Bonding.

James (1993) performed the stress analysis in rotating composite cylindrical shells. Stresses in composite cylindrical shells rotating with a constant speed about their longitudinal axis were analyzed. Each ply or ply group is treated as a separate thin layer of homogeneous and orthotropic material under the interracial stresses as surface loading. The radial stress is determined in terms of the circumferential stress through the equilibrium condition, and an average compatibility condition through the thickness of the thin layer is used.

Madhavi (2009) execute the project on design and analysis of filament wound composite pressure vessel with integrated end Domes. Material characterization of FRP of carbon T300/Epoxy for various configurations as per ASTM standards is experimentally determined using filament winding and matched die mould technique. The mechanical and physical properties thus obtained are used in the design of the composite shell. The results can be utilized to understand structural characteristics of filament wound pressure vessels with integrated end domes.

Gray (2004) developed a method to analyze filament wound composite pressure vessel using the ABAQUS finite element analysis program. They have computed the orthotropic engineering constants on an element by element basis to accurately predict the structural response of a composite overwrapped pressure vessel under internal pressure.

Aksoy (2014) performed the elastic stress analysis of laminated cylinders subjected to the mechanical, thermal and thermomechanical loads. Material properties are assumed to vary along to the radial direction. Stress and displacement distribution of the laminated cylinders are determined by using analytical method for both thermal and mechanical loads. Based on the results, materials combination plays an important role on the stresses distribution along the radius. They have presented the variation of the stresses and displacements along the radius. They have presented the calculations using MATLAB.

Bhavya (2012) studied the stress analysis with non-axisymmetric loads. A closedform solution is presented for determining the layer-by-layer stresses, strains, and displacements and first-ply failure in laminated composite cylinders subjected to nonaxisymmetric loads. The applied loads include internal and external pressure, axial force, torque, axial bending moment, uniform temperature change, rotational velocity, and interference fits. The formulation is based on the theory of anisotropic elasticity and a state of generalized plane deformation along the axis of the composite cylinder. Parametric design trade studies have been done using this closed-form solution. They have developed a computer program for performing the numerical calculations and finally presented the results from specific case studies.

Onder (2007) proposed that mechanical behavior is characterized by a set of equivalent or effective module and strength properties. Lamina properties were determined by a phenomenological approach. Analytical method, Finite element method and experimental method were applied to determine First Failure pressure of composite pressure vessel. A glass epoxy composite layer is used and are oriented symmetrically or anti symmetrically. The Tsai Wu criterion is used to compute first failure pressure of composite layers.

Pecknold (1994) studied Micromechanics-based structural analysis of thick laminated composites. The material model consists of two modules: (1) a micro-model of a unidirectional lamina, containing the basic $3 \mathrm{D}$ constitutive information for fiber and matrix constituents; and (2) a sub laminate model that enforces equilibrium of tractions between lamina, and delivers 3D homogenized stresses and strains and material tangent stiffness. This integrated approach provides the information required for evaluating damage and failure conditions at the micro structural level. 
Volety (2006) developed the Fiber Reinforced Polymer (FRP) confined concrete columns for new construction and rebuilding of concrete piers/piles in engineering structures. A 3-D finite element model of FRP confined concrete column was developed using ANSYS. Based on the FEA results, a test data base was developed taking into account all the possible ranges of the design parameters which affect the confined concrete strength.

Numerical simulation of glass-reinforced plastic cylinders under axial compression was presented by many previous authors. Madhavi (2009), Gray (2004) proposed an Analysis of a composite overwrapped Pressure Vessel by using finite element analysis program. Formulation to finite element technique and developing the failure analysis of a pressurized FRP cylinder under transverse impact loading was also presented. Experimental study and analytical modeling, failure pressure, micro mechanics based structural analysis of thick laminated composite cylinders was presented by authors Onder (2007), Pecknold (1994), Volety (2006) It is clear that the Failure analyses of FRP composite cylinders have not been investigated. There is a scope for failure analysis of FRP composite cylinders by varying the parameter $\theta$ in angle-ply laminates.

The major parameters to be considered during reinforcement of a metallic cylinder are (a) Dimensions, Length, Diameter, Thickness; (b)Material and grade of the metallic cylinder; (c) Weight of the metal cylinder; (d) Target weight of final product (Metal cylinder + Composite reinforcement); (e) Target volume constraints (maximum permissible size of metal + composite reinforcement); (f) Tolerances and surface finish requirement; (g) Painting (if necessary); (h) Operating temperature; (i) Chemical exposure (if any, including acidic, alkaline or water based environment); (j) Factor of Safety to be considered for design; (k) Maximum hoop and axial strain allowed; (l) Weight of the charge inside the cylinder; (m) Form of the charge inside the cylinder (whether in liquid or pellet form); and (n) Qualification process and parameters (testing and acceptance criteria).

\section{Formulation}

The configuration consists of a right circular cylinder of radius $\mathrm{R}$ and height $\mathrm{Z}$ rotating at a constant angular velocity $\Omega$. A cylindrical co-ordinate system with origin at the bottom of the cylinder along the axis of rotation, with radial, meridional and axial coordinates $r, \theta$, and $z$ respectively, is used. Throughout this analysis, we assume that the flow is axisymmetric, so there is no dependence on the meridional co-ordinate $\theta$. Gravitational acceleration is neglected, since it has a negligible effect for high speed gas flows. The compressible mass, momentum and energy equations in the cylindrical coordinate system are the governing equations. There are six variables in these equations, the density $\rho$, the three components of the velocity $v_{r}, v_{\theta}, v_{z}$, the pressure $p$ and the temperature $T$. The pressure is related to the density by the ideal gas equation of state,

$$
\rho=\frac{p m}{k_{B} T},
$$

where $m$ is the mass of a molecule and $k_{B}$ is the Boltzmann constant.

If the temperature on the walls of the cylinder is uniform, and there is no inflow or outflow of gas, a steady solution for the equations of motion is one where the gas undergoes solid-body rotation with angular velocity $\Omega$ at constant temperature. The temperature $\bar{T}$ and the components of the velocity $\overline{\mathbf{v}}$ in the base state in this steady state are,

$$
\bar{v}_{\theta}=\Omega r, \quad \bar{v}_{z}=0, \quad \bar{v}_{r}=0, \quad \bar{T}=T_{0},
$$

where $T_{0}$ is the constant temperature. The pressure is obtained by solving the radial 
momentum equation,

$$
\frac{d \bar{p}}{d r}=\bar{\rho} \Omega^{2} r=\frac{\bar{p} m \Omega^{2} r}{k_{B} T_{0}},
$$

where $\bar{\rho}$ is the density at steady state, together with the ideal gas equation of state,

$$
\bar{\rho}=\frac{\bar{p} m}{k_{B} T_{0}},
$$

where $m$ is the mass of a molecule and $k_{B}$ is the Boltzmann constant. The above equation is easily solved to obtain,

$$
\frac{\bar{p}}{\bar{p}_{w}}=\frac{\bar{\rho}}{\bar{\rho}_{w}}=\exp \left(-\mathcal{A}^{2}\left(1-\frac{r^{2}}{R^{2}}\right)\right),
$$

where $\bar{p}_{w}$ and $\bar{\rho}_{w}$ are the pressure and density at the wall, and $\mathcal{A}$ is the stratification parameter defined below. The total mass of gas in the cylinder is related to $\rho_{w}$ by,

$$
\text { Mass of gas }=\frac{2 \pi R^{2} Z \bar{\rho}_{w}\left(1-\exp \left(-\mathcal{A}^{2}\right)\right)}{2 \mathcal{A}^{2}},
$$

where $Z$ is the total height of the cylinder.

It is useful to discuss the different dimensionless numbers used in the analysis. The stratification parameter obtained from the equation for the density is,

$$
\mathcal{A}=\sqrt{\frac{m \Omega^{2} R^{2}}{2 k_{B} T_{0}}} \propto \frac{R \Omega}{c_{s}},
$$

where $(R \Omega)$ is the characteristic flow velocity, and $c_{s}=\sqrt{\gamma k_{B} T_{0} / m}$ is the speed of sound. The Reynolds number, used a little later, is defined as

$$
\operatorname{Re}=\frac{\rho_{w} \Omega R^{2}}{\bar{\mu}},
$$

where $\bar{\mu}$ is the viscosity of the fluid in the unperturbed state. The viscosity of a dilute gas scales as,

$$
\bar{\mu} \sim \frac{\sqrt{m k_{B} T_{0}}}{d^{2}}
$$

where $m$ is the molecular mass and $d$ is the molecular diameter. Using the above expression for the viscosity, it can easily be verified that the Reynolds number can be rewritten as,

$$
\operatorname{Re} \sim \frac{\mathcal{A}}{\mathrm{Kn}},
$$

where the Knudsen number is defined as

$$
\mathrm{Kn}=\left(m / \sqrt{2} \pi \rho_{w} d^{2} R\right)=(\lambda / R),
$$

where $\lambda=\left(m / \sqrt{2} \pi \rho_{w} d^{2}\right)$ is the mean free path at the wall. The other dimensionless groups used are the Prandtl number and the ratio of specific heats,

$$
\begin{gathered}
\operatorname{Pr}=\frac{C_{p} \bar{\mu}}{\bar{K}}, \\
\gamma=\frac{C_{p}}{C_{v}},
\end{gathered}
$$

which are both $O(1)$ for an ideal gas. 


\subsection{The separation factor}

The basic reason for separation of isotopes in a high speed rotating cylinder is that the pressure gradient of the gas is dependent on its molecular weight. Thus different isotopes have different pressure gradients and separation occurs in a way, that the light isotope has a higher concentration in the centre than at the wall. Between the peripheri $(r=R)$ and axis $(r=0)$ for two different gases, we have

$$
\frac{\bar{p}_{1, R}}{\bar{p}_{2, R}}=\frac{\bar{p}_{1,0}}{\bar{p}_{2,0}} \exp \left(\frac{\Delta m \Omega^{2} R^{2}}{2 k_{B} T_{0}}\right) .
$$

In a binary gas mixture, if $\bar{N}_{1}$ is the mole fraction of the component- 1 having molecular mass $m_{1}$, then

$$
\begin{aligned}
\bar{p}_{1}+\bar{p}_{2} & =\bar{p}, \\
\bar{N}_{1} & =\frac{\bar{p}_{1}}{\bar{p}}, \\
1-\bar{N}_{1} & =\frac{\bar{p}_{2}}{\bar{p}} .
\end{aligned}
$$

Then 2.14 becomes,

$$
\left(\frac{\bar{N}_{1}}{1-\bar{N}_{1}}\right)_{R}=\left(\frac{\bar{N}_{1}}{1-\bar{N}_{1}}\right)_{0} \exp \left(\frac{\Delta m \Omega^{2} R^{2}}{2 k_{B} T_{0}}\right),
$$

and accordingly the radial separation factor,

$$
\begin{aligned}
\alpha_{0} & =\exp \left(\frac{\Delta m \Omega^{2} R^{2}}{2 k_{B} T_{0}}\right), \\
& =\exp \left(\epsilon \mathcal{A}^{2}\right),
\end{aligned}
$$

where $\epsilon$ is defined as $\epsilon=\Delta m / m_{a v}$.

In a countercurrent gas centrifuge the actual separation factor can be enlarged to several times that of the radial separation factor by establishing an axial countercurrent. The enhancement factor depends on the aspect ratio $(Z / 2 R)$. In case of very low throughput the axial separation factor will approach the value,

$$
\begin{aligned}
\alpha_{\text {axial }} & =\alpha_{0}^{\sqrt{2} \frac{Z}{2 R}}, \\
& =\exp \left(\epsilon \mathcal{A}^{2} \sqrt{2} \frac{Z}{2 R}\right) .
\end{aligned}
$$

\subsection{The separative power}

The performance of a gas centrifuge is measured in terms of separative power and is expressed in kilogram of separative work units per year ( $k g S W U / y e a r)$. The separative power is the product of the mass flows of a separation element multiplied with its concentration in the form of a value function $(V)$ and defined as,

$$
\delta U=P V\left(N_{P}\right)+W V\left(N_{W}\right)-F V\left(N_{F}\right),
$$

where $P, W$, and $F$ are the product, waste, and feed flow rates. $N_{P}, N_{W}$ and $N_{F}$ are the respective concentrations. The value function is defined as,

$$
V(N)=(2 N-1) \log _{e} \frac{N}{1-N}
$$


The ratio of product flow to feed flow is defined as 'cut' $(\theta)$,

$$
\theta=\frac{P}{F} \text {. }
$$

Therefore, the separative power can be written as,

$$
\delta U=F\left(\theta V\left(N_{P}\right)+(1-\theta) V\left(N_{W}\right)-V\left(N_{F}\right)\right) .
$$

If the definition of separative power is applied to a volume element inside the gas centrifuge and its diffusive flows, then under ideal condition as upper limit the maximum separative power according to Dirac (Cohen (1951)) can be derived as follows. The separative power $(\delta U)$ for a small slab of thickness $d s$, with faces $d \sigma$ normal to $\nabla N$ and sides parallel to $\nabla N$, is given by (Cohen (1951)),

$$
d U=\frac{p D}{k_{B} T} \frac{\left(\Delta m \frac{\Omega^{2} r \mathbf{e}_{r}}{k_{B} \bar{T}}(N(1-N))-\nabla N\right)}{N^{2}(1-N)^{2}} d \sigma d s .
$$

Taking $\nabla N$ as a variable quantity and $d \sigma$, and everything else constant, 2.23 is maximum when,

$$
\nabla N=\frac{1}{2} \Delta m \frac{\Omega^{2} r \mathbf{e}_{r}}{k_{B} \bar{T}}(N(1-N))
$$

that is, along the direction of centrifugal field, and one-half the equilibrium value. Then we have,

$$
d U_{M A X}=\frac{p D}{k_{B} T}\left(\frac{\Delta m \Omega^{2} r \mathbf{e}_{r}}{2 k_{B} \bar{T}}\right)^{2} d \sigma d s .
$$

Integrating over the whole length $Z$ and radius $R$,

$$
\begin{aligned}
\delta U_{M A X} & =\int d U_{M A X}, \\
& =\frac{p D}{k_{B} T}\left(\frac{\Delta m \Omega^{2}}{2 k_{B} \bar{T}}\right)^{2} \int_{0}^{R} r^{2} 2 \pi r d r Z, \\
& =\frac{\pi}{2} \rho D\left(\frac{\Delta m \Omega^{2} R^{2}}{2 k_{B} \bar{T}}\right)^{2} Z, \\
& =\frac{\pi}{2} \rho D\left(\epsilon \mathcal{A}^{2}\right)^{2} Z .
\end{aligned}
$$

Here, $\delta U$ is the separative power, $D$ is the gaseous diffusion coefficient, $\rho$ is the gas density, $\mathcal{A}$ is the stratification parameter, and $Z$ is the total height of the cylinder.

The above relation has been derived under the assumptions that the separation is under isothermal condition, there is no axial back diffusion, and that the radial concentration gradient is half its equilibrium value.

The above equation suggests that increase in length, increases the separative power output linearly, and increase in speed with the $4^{\text {th }}$ power. The actual separative power obtained from a centrifuge is less that the maximum value, and therefore the separative power is expressed as,

$$
\delta U=\epsilon_{o} \times \delta U_{M A X},
$$

where, $\epsilon_{o}$ is the overall efficiency of the centrifuge and is a complex function of speed, length, wall pressure, temperature, secondary flow generation, cut, and internal geometric centrifuge data. However generally improvements in separative power can be obtained by (a) Increase in speed, (b) Increase in length, and (c) Increase in efficiency. 


\begin{tabular}{|c|c|c|c|c|}
\hline Material & $\rho$ & $\sigma$ & E & $v_{\theta, \max }$ \\
\hline Special Steel & 8000 & 2200 & $2.0 \times 10^{11}$ & 524.40 \\
\hline Carbon Fiber (T-700G) & 1800 & 2550 & $2.95 \times 10^{11}$ & 1190.23 \\
\hline Carbon Fiber (T-1000G) & 1800 & 3040 & $2.95 \times 10^{11}$ & 1299.57 \\
\hline
\end{tabular}

TABle 1. The Density $\rho\left(\mathrm{kg} / \mathrm{m}^{3}\right)$, Tensile strength $\sigma(M P a)$, Young Modulus $E(P a)$, and maximum tangential speed $v_{\theta, \max }(\mathrm{m} / \mathrm{sec})$ of Special Steel, Carbon Fiber (T-700G), and Carbon Fiber (T-1000G) at temperature $300 \mathrm{~K}$.

\subsection{The maximum peripheral speed}

Consider a cylindrical shell of radius $r$ and thickness $d r$, made of material of density $\rho$ and rotating at angular velocity $\omega \mathrm{rad} / \mathrm{sec}$. We take a volume element of the shell of height $d z$ subtending an angle $d \theta$. The mass of the element is

$$
d m=\rho r d r d z d \theta
$$

This mass experiences a centrifugal force in the outward $r$ direction,

$$
\omega^{2} r d m=\rho \omega^{2} r^{2} d r d z d \theta .
$$

This must be balanced by the components in the opposite direction, $\sigma_{\theta} \sin (d \theta / 2)$, of the tangential stresses $\sigma_{\theta}$ acting on the two surfaces $d r d z$ offset by angle $d \theta$,

$$
2 \sigma_{\theta} \sin \left(\frac{d \theta}{2}\right) d r d z=\rho \omega^{2} r^{2} d r d z d \theta \text {. }
$$

For the small value of $d \theta$, we obtain

$$
\begin{aligned}
\sigma_{\theta} & =\rho \omega^{2} r^{2}, \\
& =\rho v_{\theta \max }^{2},
\end{aligned}
$$

where $v_{\theta \max }$ is the maximum tangential speed, at which the tangential stress reaches the tensile strength of the material.

The density $(\rho)$, tensile strength $(\sigma)$, modulus of elasticity $(E)$, and maximum tangential speed $\left(v_{\theta, \max }\right)$ of Special Steel, Glass Fiber, Carbon Fiber (T-700G), and Carbon Fiber (T-700G) are give in table 1.

\subsection{The resonant vibrations}

Certain angular velocities $\left(\Omega_{i}\right)$ causes a thin, hollow cylinder to go into resonant longitudinal vibrations. If a high speed rotor is driven for any length of time at or near one of these angular velocities, rotational energy is used to increase the amplitude of longitudinal vibrations until the rotor or its bearings may be wrecked. Therefore, it is important to avoid tangential speeds at which a high speed rotor of given length and radius will be in resonance. From vibrational analysis the longitudinal vibration frequency $\Omega_{i}$ of a thin hollow cylinder of radius $R$, modulus of elasticity $E$, and length $Z$, unrestrained at the ends, in the $i^{t h}$ mode is

$$
\Omega_{i}=\lambda_{i} \sqrt{\frac{E}{2 \rho}} \frac{R}{Z^{2}}
$$


where $\lambda_{i}$ are the eigenvalues, and $i$ is the number of loops into which the profile of the cylinder is displaced. Since,

$$
v_{\theta, i}=\Omega_{i} r
$$

the length to radius ratio at which rotors of each of the materials run at maximum tangential speed would be in resonant vibration is,

$$
\begin{aligned}
\left(\frac{Z}{R}\right)_{i} & =\sqrt{\frac{\lambda_{i}}{v_{\theta, \max }}}\left(\frac{E}{2 \rho}\right)^{\frac{1}{4}}, \\
& =\sqrt{\lambda_{i}}\left(\frac{E}{2 \sigma}\right)^{\frac{1}{4}} .
\end{aligned}
$$

Rotors that are shorter than the first critical length are said to be the subcritical rotors. Such rotors do not need special means to avoid resonant speeds. Rotors that are longer than the first critical length are called supercritical. They must be operated at speeds away from resonance and must be provided with drives of sufficient power to accelerate them quickly through resonant speed.

\subsection{The rotational radial load}

The main difficulty in spinning rotors at high speed is the engineering impossibility of making a perfectly balanced rotor, one in which the geometric and mass axes coincide. The net unbalance is usually measured either in units of $m_{1} R$, the balance mass required at a given radius of application, or $\mathcal{M}$, the rotor mass $\mathcal{M}$ multiplied by the distance $\Delta$ of the center of gravity from the geometric center.

The rotational radial load or unbalance force is given by,

$$
\begin{gathered}
F_{r}=m_{1} R \Omega^{2}, \\
\mathcal{M} \cdot \otimes \epsilon,(2.35)
\end{gathered}
$$

where the displacement $\Delta$ is given by,

$$
\Delta=\frac{e R}{2 t}
$$

Here, $e$ is the eccentricity of manufacture, and $t$ is the rotor thickness.

\subsection{The critical frequencies}

The natural cylindrical frequency of a spinning rotor is given by the standard solution for simple harmonic motion,

$$
f_{1}=\left(\frac{2 S}{\mathcal{M}}\right)^{1 / 2} .
$$

These natural cylindrical whirls can be excited at rest or at any frequency of rotation of the shaft, but will always occur at the frequency given by the above equation.

The critical frequencies defined as, at which the rotational frequency equals the frequencies of the natural modes of vibration. At these frequencies, sometimes called critical speeds, the amplitude of rotor whirl can attain dangerous proportions. The exact frequencies of the criticals can be obtained by solving for the intersection points, giving the frequencies of the cylindrical critical speeds as,

$$
f_{1}=\left(\frac{2 S}{\mathcal{M}}\right)^{1 / 2} .
$$




\subsection{Calculation of flexural critical speeds}

There are both analytical and numerical methods for calculating these frequencies. The calculations, if done exactly, should also allow for any deflection of the rotor due to shearing forces and for the effects of the gyroscopic couples of the whirling sections. However, the physical principles are best understood by ignoring these small effects and using the method developed by Lord Rayleigh (1877). This method is to equate the potential energy of the thin tube when at its maximum amplitude with its maximum kinetic energy, which occurs when passing through the unbent condition. The Rayleigh theory, gives the solution for the peripheral velocity at the critical speeds as,

$$
V_{n}=\frac{\left(n+\frac{1}{2}\right)^{2} \Pi^{2}}{4(Z / D)^{2}}\left(\frac{E}{2 \rho}\right)^{1 / 2} .
$$

This standard Rayleigh equation gives the critical speeds for a given $(Z / D)$ ratio or, by transposing the equation, the $(Z / D)$ ratio for a given critical speed. The critical frequencies and $(Z / D)$ ratios for thin-walled tubes are independent of thickness but dependent on the specific modulus $(E / \rho)$ of flexure of the material of construction.

\subsection{Bond strength}

The bonding between reinforcement and matrix is critical to the performance of the composite material. A strong bond between reinforcement and matrix is desired so that the loads can be transferred efficiently from the matrix to the reinforcement.

The reinforcement/matrix interactions involves the recognition of a flexible, threedimensional interphase which is thought to exist between the matrix and the reinforcement. This interphase is a polymer network formed by the coupling compound or the size and into which the resin or matrix can penetrate. The network have chemical attachments to the fiber surface, but the main purpose of the network is to provide a lattice which the matrix molecules can be penetrate and can be held in close proximity to the fibers. Therefore, the interphase is a region where size and matrix have diffused into each others' domain. There also be selective adsorption of resin on to the fiber. The interphase is responsible for transferring the load from matrix to the fiber.

Although the interphase has lower modulus and lower strength than either the fiber or the matrix, true cohesive failure in tension is quite rare within the main part of the interphase zone.

Predictions of bond strength between the interphase and the reinforcement is obtained by applying the general principle of surface chemistry. If the forces on a molecule in the bulk are compared to the forces on a molecule at the surface, the forces on the bulk molecules cancel whereas the forces on the surface molecules are unbalanced. This unbalance gives rise to a surface free energy $\gamma$, which is defined as the energy necessary to form a unit area of new surface or the energy necessary to move a molecule from the bulk to the surface. The work of adhesion, $W_{A}$, is defined as the work required to separate two particles and is, in terms of the surface free energy, $\gamma$,

$$
W_{A}=\gamma_{A}+\gamma_{B}-\gamma_{A B},
$$

that is, the work of adhesion is the surface free energy of particle $A$ plus the surface free energy of particle $B$ minus the surface free energy of the interaction between $A$ and $B$.

\subsection{Properties from micromechanics}

Some basic properties is estimated using 'property-structure' relationships, or micromechanics. The basic assumptions are : (a) The composite ply is macroscopically homogeneous and linearly elastic; (b)the fibers are linearly elastic and homogeneous; (c) the 
matrix is linearly elastic and homogeneous; (d) both the fiber and matrix are free of voids; (e) the interface is completely bonded, and there is no transitional region between the matrix and reinforcement; (f) the mechanical properties of the individual constituents are the same whether they are made by themselves or made up within the composite.

With this in mnd, the values of $E_{11}$ (longitudinal modulus), $\nu_{12}$ (principle Poisson's ratio), and $\alpha_{11}$ (principle thermal expansion coefficient) is expressed in terms of the matrix/fiber properties themselves and the volumefraction of the respective ingredients. These expressions are derived from the rule of mixtures theory and results in

$$
\begin{gathered}
E_{11}=V_{f} E_{f}+V_{m} E_{m}, \\
\nu_{12}=V_{f} \nu_{f}+V_{m} \nu_{m}, \\
\alpha_{12}=V_{f} \alpha_{f}+V_{m} \alpha_{m},
\end{gathered}
$$

where $V_{f}$ and $V_{m}$ represent the fiber and matrix volume percentages, and the values of $E_{f}, E_{m}, \nu_{f}, \nu_{m}, \alpha_{f}$, and $\alpha_{m}$ are the individual constituent properties for tensile modulus, Poisson's ratio, and thermal expansion coefficient of the fiber and matrix.

\subsection{Fraction of load carried by fibers}

$$
\begin{aligned}
\frac{P_{f}}{P_{c}} & =\frac{\sigma_{f} A_{f}}{\sigma_{c} A_{c}}, \\
& =\frac{E_{f} \epsilon_{f} A_{f}}{E_{c} \epsilon_{c} A_{c}}, \\
& =\frac{E_{f}}{E_{c}} v_{f},
\end{aligned}
$$

where $v_{f}$ is the volume fraction of the fibers in the composite. In the present analysis $v_{f}$ is taken as 0.6 (60\% fiber volume).

\subsection{Elastic strain energy}

If the stress tensor $\sigma$, having components $\sigma_{i j}$, is decomposed into two tensors $\sigma^{\prime}$ and $\sigma^{\prime \prime}$, whose components respectively are $\sigma_{i j}^{\prime}$, and $\sigma_{i j}^{\prime \prime}$, it results,

$$
\sigma_{i j}=\sigma_{i j}^{\prime}+\sigma_{i j}^{\prime \prime},
$$

and the elastic energy is expressed in the form,

$$
\begin{aligned}
U & =\frac{1}{2} \sigma_{i j} \epsilon_{i j}, \\
& =\frac{1}{2}\left(\sigma_{i j}^{\prime}+\sigma_{i j}^{\prime \prime}\right)\left(\epsilon_{i j}^{\prime}+\epsilon_{i j}^{\prime \prime}\right) \\
& =\frac{1}{2} \sigma_{i j}^{\prime} \epsilon_{i j}^{\prime}+\frac{1}{2} \sigma_{i j}^{\prime \prime} \epsilon_{i j}^{\prime \prime}+\frac{1}{2}\left(\sigma_{i j}^{\prime} \epsilon_{i j}^{\prime \prime}+\sigma_{i j}^{\prime \prime} \epsilon_{i j}^{\prime}\right)
\end{aligned}
$$

\subsection{Stress analysis}

There are two important requirements of the centrifuge rotor so far as stress analysis is concerned. These are that (a) the stress levels of the rotor components be no greater than the acceptable working stress of the material of construction, and (b) the strain levels of the component be such that individual parts do not become loose at speed.

It is therefore necessary to perform a detailed stress analysis of any rotating system to ensure that these conditions are met. The theory of stress analysis forms part of 


\begin{tabular}{|c|c|c|c|c|}
\hline Material & $\rho$ & $\sigma$ & E & $v_{\theta, \max }$ \\
\hline Special Steel & 8000 & 2200 & $2.0 \times 10^{11}$ & 524.40 \\
\hline Carbon Fiber (T-700G) & 1800 & 2550 & $2.95 \times 10^{11}$ & 1190.23 \\
\hline Carbon Fiber (T-1000G) & 1800 & 3040 & $2.95 \times 10^{11}$ & 1299.57 \\
\hline
\end{tabular}

TABle 2. The Density $\rho\left(k g / \mathrm{m}^{3}\right)$, Tensile strength $\sigma(M P a)$, Young Modulus $E(P a)$, and maximum tangential speed $v_{\theta, \max }(\mathrm{m} / \mathrm{sec})$ of Special Steel, Carbon Fiber (T-700G), and Carbon Fiber (T-1000G) at temperature $300 \mathrm{~K}$.

the science of elasticity in the design of the centrifuge rotor. Here we will discuss the the important features relevant to the stresses in rotating cylinders and discs, the two important components part of the centrifuge rotor.

Consider a cylindrical shell of radius $r$ and thickness $d r$, made of material of density $\rho$ and rotating at angular velocity $\omega \mathrm{rad} / \mathrm{sec}$. We take a volume element of the shell of height $d z$ subtending an angle $d \theta$. The mass of the element is

$$
d m=\rho r d r d z d \theta
$$

This mass experiences a centrifugal force in the outward $r$ direction,

$$
\omega^{2} r d m=\rho \omega^{2} r^{2} d r d z d \theta .
$$

This must be balanced by the components in the opposite direction, $\sigma_{\theta} \sin (d \theta / 2)$, of the tangential stresses $\sigma_{\theta}$ acting on the two surfaces $d r d z$ offset by angle $d \theta$,

$$
2 \sigma_{\theta} \sin \left(\frac{d \theta}{2}\right) d r d z=\rho \omega^{2} r^{2} d r d z d \theta .
$$

For the small value of $d \theta$, we obtain

$$
\begin{aligned}
\sigma_{\theta} & =\rho \omega^{2} r^{2}, \\
& =\rho v_{\theta}^{2} \underset{\text { max }}{2},
\end{aligned}
$$

where $v_{\theta \max }$ is the maximum tangential speed, at which the tangential stress reaches the tensile strength of the material. This equation shows that the peripheral speed of a centrifuge is fixed only by the specific tensile strength of the material of construction.

The density $(\rho)$, tensile strength $(\sigma)$, modulus of elasticity $(E)$, and maximum tangential speed $\left(v_{\theta, \max }\right)$ of Special Steel, Glass Fiber, Carbon Fiber (T-700G), and Carbon Fiber (T-700G) are give in table 2 .

The radial and axial strains depend on the specific modulus and the Poisson ratio of the material of construction according to the equations

$$
\begin{gathered}
\epsilon_{r}=\left(\rho V^{2}\right) / E, \\
\epsilon_{a}=\left(\nu \rho V^{2}\right) / E .
\end{gathered}
$$

Here, $E$ is the Young's modulus, and $\nu$ is the Poisson ratio. The general result is that the stress levels of discs are much lower than those of tubes made of the same material and spinning at the same peripheral speed. This is because a disc can provide inward radial forces.

The most important general result is that the maximum peripheral speed that can be 
attained by various tubes and discs is in every case limited by the strength-to-weight ratio of the material of construction.

\subsection{Elastic stress analysis of laminated cylinders}

Next, we have performed the elastic stress analysis of laminated cylinders subjected to the mechanical, thermal and thermomechanical loads. Material properties are assumed to vary along the radial direction.

The governing differential equations of equilibrium for cylinders in 2D are,

$$
\begin{gathered}
\frac{\partial \sigma_{r}}{\partial r}+\frac{1}{r} \frac{\partial \tau_{r \theta}}{\partial \theta}+\left(\frac{\sigma_{r}-\sigma_{\theta}}{r}\right)+R=0, \\
\frac{\partial \tau_{r \theta}}{\partial r}+\frac{1}{r} \frac{\partial \sigma_{\theta}}{\partial \theta}+\frac{2 \partial \tau_{r \theta}}{r}+R=0 .
\end{gathered}
$$

Stress components are independent of $\theta$ because of the symmetry and can be only functions of $r$. Also, the shear stress, $\tau_{r \theta}$, equals to zero. Hence, with centrifugal force the above equations becomes,

$$
\frac{\partial \sigma_{r}}{\partial r}+\left(\frac{\sigma_{r}-\sigma_{\theta}}{r}\right)+\rho \Omega^{2} r=0,
$$

where $\sigma_{r}$ and $\sigma_{\theta}$ are radial and circumferential stresses, respectively. $\rho$ is the density of the material in the radial direction and $\Omega$ is the angular velocity.

For plain strain case, the relation between strain and stress under the temperature can be described by Hookes Law,

$$
\begin{aligned}
& \epsilon_{r}=\frac{1+\nu}{E(r)}\left((1-\nu) \sigma_{r}-\nu \sigma_{\theta}+E(r) \alpha(r) T(r)\right), \\
& \epsilon_{\theta}=\frac{1+\nu}{E(r)}\left((1-\nu) \sigma_{\theta}-\nu \sigma_{r}+E(r) \alpha(r) T(r)\right),
\end{aligned}
$$

where $\nu, E, \alpha$, and $T$ are Poissons ratio, elasticity modulus, thermal expansion coefficient and temperature, respectively.

Strain-displacement relations are the following;

$$
\begin{gathered}
\epsilon_{r}=\frac{d u}{d r} \\
\epsilon_{\theta}=\frac{u}{r},
\end{gathered}
$$

where $u$ is the displacement component in the radial direction. The compatibility equation can be expressed as,

$$
\epsilon_{r}=\frac{d}{d r}\left(r \epsilon_{\theta}\right) .
$$

Stress components for rotating cylinder in terms of the stress function can be described as

$$
\begin{gathered}
\sigma_{r}=\frac{F}{r}, \\
\sigma_{\theta}=\frac{d F}{d r}+\rho \Omega^{2} r^{2} .
\end{gathered}
$$

Applying the above equations into the relation between strain and stress and then sub- 
stituted into the compatibility equation gives

$$
r^{2} \frac{d^{2} F}{d r^{2}}+r \frac{d F}{d r}-F=-\rho \Omega^{2} r^{3}\left(\frac{\nu}{1-\nu}+3\right)-\frac{E(r) \alpha(r)}{1-\nu} r^{2} T(r) .
$$

The above equation is the second order differential equation and applying $r=e^{t}$ transformation yields

$$
\frac{d^{2} F}{d r^{2}}-F=-\rho \Omega^{2} e^{3 t}\left(\frac{\nu}{1-\nu}+3\right)-\frac{E(r) \alpha(r)}{1-\nu} e^{2 t} T(r) .
$$

\subsubsection{Analysis for mechanical loading}

For a rotating cylinder which has an angular velocity $(\Omega)$, the above equation reduces to

$$
\frac{d^{2} F}{d r^{2}}-F=-\rho \Omega^{2} e^{3 t}\left(\frac{\nu}{1-\nu}+3\right) .
$$

The above equation can be solved for the stress function, $F$, and can be written as

$$
F=C_{1} r+\frac{C_{2}}{r}+A r^{3}
$$

where $A$ is expressed as,

$$
A=-\frac{\rho \Omega^{2}\left(\frac{\nu}{1-\nu}+3\right)}{6}
$$

and the stress components are

$$
\begin{gathered}
\sigma_{r}=\frac{F}{r}=C_{1}+\frac{C_{2}}{r^{2}}+A r^{2} \\
\sigma_{\theta}=\frac{d F}{d r}+\rho \Omega^{2} r^{2}=-\frac{C_{2}-r^{2} C_{1}-3 r^{4} A}{r^{2}}+\rho \Omega^{2} r^{2}
\end{gathered}
$$

To determine $C_{1}$ and $C_{2}$, the boundary conditions are,

$$
\sigma_{r}=0, r=r_{i}, \text { and } \sigma_{r}=0, r=r_{o},
$$

where $r_{i}$ and $r_{o}$ are the inner and outer radius of laminated cylinders, respectively. By using these boundary conditions,

$$
\begin{gathered}
C_{1}=\frac{\rho \Omega^{2}\left(r_{o}^{2}+r_{i}^{2}\right)(2 \nu-3)}{6(\nu-1)}, \\
C_{2}=-\left(\frac{\left(\rho \Omega^{2} r_{o}^{2} r_{i}^{2}\right)(2 \nu-3)}{6(\nu-1)}\right) .
\end{gathered}
$$

\subsubsection{Analysis for thermal loading}

Under the radially linearly-increasing temperature which is,

$$
T=T_{o}\left(\frac{r_{o}+r}{r_{o}-r_{i}}\right)
$$

we have

$$
\frac{d^{2} F}{d r^{2}}-F=-\frac{E(r) \alpha(r)}{1-\nu} e^{2 t} T(r)
$$

The above equation can be solved for the stress function, $F$, and can be written as

$$
F=C_{1} r+\frac{C_{2}}{r}+S r^{2}
$$


where $S$ is expressed as,

$$
S=\frac{E \alpha T_{0}}{3(1-\nu)\left(r_{o}-r_{i}\right)},
$$

and the stress components are

$$
\begin{gathered}
\sigma_{r}=\frac{F}{r}=C_{1}-\frac{C_{2}}{r^{2}}+S r, \\
\sigma_{\theta}=\frac{d F}{d r}=C_{1}+\frac{C_{2}}{r^{2}}+2 S r .
\end{gathered}
$$

To determine $C_{1}$ and $C_{2}$, the boundary conditions are,

$$
\sigma_{r}=0, r=r_{i}, \text { and } \sigma_{r}=0, r=r_{o},
$$

where $r_{i}$ and $r_{o}$ are the inner and outer radius of laminated cylinders, respectively. By using these boundary conditions,

$$
\begin{aligned}
C_{1} & =\frac{E T_{0} \alpha\left(r_{o}^{2}+r_{i}^{2}+r_{o} r_{i}\right)}{3\left(r_{o}^{2}-r_{i}^{2}\right)(\nu-1)}, \\
C_{2} & =-\frac{E T_{0} \alpha\left(r_{o}^{2}+r_{i}^{2}\right)}{3\left(r_{o}^{2}-r_{i}^{2}\right)(\nu-1)} .
\end{aligned}
$$

\subsubsection{Analysis for thermo-mechanical loading}

For rotating cylinder subject to the radially linearly-increasing temperature is solved for the stress function, F, and can be written as

$$
F=C_{1} r+\frac{C_{2}}{r}+K r^{3}+L r^{2},
$$

where $K$ and $L$ are expressed as,

$$
\begin{aligned}
& K=-\frac{\rho \Omega^{2}\left(\frac{\nu}{1-\nu}+3\right)}{8}, \\
& L=\frac{E \alpha T_{0}}{3(1-\nu)\left(r_{o}-r_{i}\right)},
\end{aligned}
$$

and the stress components are

$$
\begin{gathered}
\sigma_{r}=\frac{F}{r}=C_{1}+\frac{C_{2}}{r^{2}}+K r^{2}+L r \\
\sigma_{\theta}=\frac{d F}{d r}+\rho \Omega^{2} r^{2}==C_{1}-\frac{C_{2}}{r^{2}}+3 K r^{2}+2 L r+\rho \Omega^{2} r^{2} .
\end{gathered}
$$

To determine $C_{1}$ and $C_{2}$, the boundary conditions are,

$$
\sigma_{r}=0, r=r_{i}, \text { and } \sigma_{r}=0, r=r_{o},
$$

where $r_{i}$ and $r_{o}$ are the inner and outer radius of laminated cylinders, respectively.

\subsection{Magnetic stresses}

In the past few decades, the use of composite materials in engineering application increases rapidly. Therefore,the stress analysis of layered media has been investigated by earlier researchers. Due to the necessity in considering the boundary conditions on each of the multiple interfaces for such a problem, the solving process becomes more complicated as comparing with the homogeneous counterpart. Thus the stress analysis of 
multi-layered media problem results in finding the solution for a system of simultaneous equations with a lot of unknown constants as derived by Iyengar and Alwar [1] and Chen [2]. Nevertheless, several methods have been developed to provide an effficient approach in studying the elastic problem of multi-layered media. Such as Bulfer [3] used the method of transfer matrix which is expressed in terms of the infinite series expansion and can be solved with various orders of approximation, Lin and Keer [4] applied the flexibility matrix approach with the boundary integral formulation to solve the elastic problem of a vertical crack in a multi-layered medium. Based upon the alternating technique, Choi and Earmme [5] conducted the stress analysis of the singularity problem in an isotropic plane layered trimaterial. Comparing with the aforementioned studies of straight interfaces, the corresponding problem of circular interfaces involve more mathematical complexity. Several investigators considered the elastic problem of a three-phase cylinder. As denoted by Benveniste et al.[6], a three-phase circular boundary problem with perfect interfaces can be transformed into a corresponding two-phase problem with imperfect interface by letting the thickness of intermediate phase tends to zero. Luo [7]employed the Laurent series expansion and the method of analytical continuation to find a solution for the elastic problem of three-phase composite cylinder with an edge dislocation at the intermediate annular region. By the use of heterogenization technique, Honein et al.[8]considered the anti-plane elastostatics of circularly cylindrical and plane layered media. Chao et al.[9] used the alternating technique with analytic continuation to obtain the stress evoked by a point force. Due to the possible application of a structure in a magnetic environment, the interaction between the magnetic field and the deformation of the structure is a relevant concern. Especially in an environment of strong magnetic field, such as magnetic fusion reactor and magnetic levitated vehicles, the analysis of magnetoelastic interaction is necessary in considering the safety of structure. Just like that in the pure elastic case, the application of composite material is also competent in the magnetoelastic one. For the problem associated with inhomogeneities in magnetoelasticity, Maugin et al.[10] presented a theoretic formulation of nonlinear anisotropic inhomogeneous electromagnetoelasticity. Lin [11] used the technique of conformal mapping and the method of analytical continuation to find the magnetic and the magnetoelastic fields on both the matrix and a perfectly bonded elliptic inclusion. Based upon the complex variable theory and the method of analytical continuation,Lin and Chen [12] found the magnetoelastic stresses on a circular shell subjected to a point magnetic source or a remote uniform magnetic induction. By applying the Laurent series expansion and expanding the definition of Airy s stress function to magnetoelasticity, Chen and Lin [13]obtained the magnetoelastic fields for an infinite matrix with multiple circular inclusions.

\subsection{Creep properties}

In the present engineering application the component made of composite materials has to operate under severe thermo-mechanical loading where creep becomes significant. Therefore, the present investigation also deals with the creep behavior of composite materials. The progressive deformation of a material at constant load is called creep. To determine the engineering creep curve of a metal, a constant load is applied to a standard specimen maintained at a constant temperature and the strain (extension)is determined as a function of time.

The first stage of the creep, known as primary creep, represents a region of decreasing creep rate. Primary creep is a period of predominantly transient creep in which the resistance of the material increases by virtue of its own deformation. Primary creep occurs at low temperature and low stress levels. The second stage of creep, also known as secondary creep, is a period of nearly constant creep rate which results from a balance between the 
competing processes of strain hardening and ecovery. For this reason, secondary creep is usually referred to as steady state creep.The average value of the creep rate during secondary creep is called the minimum creep rate. Third stage or tertiary creep mainly occurs in constant load creep tests at high stresses and high temperature. Tertiary creep occurs when there is an effective reduction in cross sectional area eithe because of necking or internal void formation. Third stage creep is often associated with metallurgical changes such as coarsening of precipitate particles, recrystallization or diffusional changes in the phases present. The creep curve can be represented by the following equation,

$$
\epsilon=\epsilon_{0}+\epsilon_{t}\left(1-e^{-r t}\right)+\epsilon_{s}
$$

where, $\epsilon$ is total strain, $\epsilon_{0}$ is the instantaneous strain on loading, $\epsilon_{t}$ is the limit for transient creep rate to the transient creep strain, and $\epsilon_{s}$ is the steady state creep rate.

Creep analysis of rotating cylinder subjected to elevated temperature has been the subject of numerous investigations. In most of the studies the cylinder material is assumed to be monolithic and only very limited studies deal with the behavior of a rotating cylinder made of composite material. The efforts have been made to develop mathematical models to evaluate creep response of a rotating cylinder. The excellent mechanical properties like high specific strength and stiffness, and high temperature stability offered by aluminium/aluminium alloy based composites consisting of ceramic reinforcement (e.g. Sic) make them appropriate choice for use in severe thermo-mechanical loading conditions as observed in some of the applications of rotating cylinders.

Further, the creep performance of engineering components such as rotating disc made of composites can be significantly improved by modifying the materials parameters like reinforcement size, shape and content, and the temperature of application [24]. In the light of above said it is decided to investigate effect of material parameters on the steadystate creep behavior of a rotating thick cylinder made of Al-SiCp. The following objectives are set for the present work:

a) develop a mathematical model to evaluate steady state creep response of rotating cylinder, b) development of computer code to calculate stresses and strain rates in cylinder, c) investigate the effect of reinforcement size and content, and operating temperature on the creep behavior of rotating cylinder.

In order to investigate the effect of material parameters on the steady state creep performance of a hollow rotating cylinder, a mathematical model is developed. The model will be used to calculate stress and strain-rate distributions in the wall of a hollow thinwalled circular cylinder, rotating about its own axis with a constant angular speed.

The equation of equilibrium for a rotating cylinder is given by the relation

$$
\frac{\partial \sigma_{r}}{\partial r}+\left(\frac{\sigma_{r}-\sigma_{\theta}}{r}\right)+\rho \Omega^{2} r=0
$$

where $\sigma_{r}$ and $\sigma_{\theta}$ are radial and circumferential stresses, respectively. $\rho$ is the density of the material in the radial direction and $\Omega$ is the angular velocity.

It is assumed that the creep deformation does not involve appreciable volume changes thus,

$$
\epsilon_{r}+\epsilon_{\theta}+\epsilon_{z}=0
$$

If the cylinder is constrained from contracting in axial direction, then,

$$
\epsilon_{z}=0
$$

Therefore,

$$
\epsilon_{r}+\epsilon_{\theta}=0
$$


The material of the rotating cylinder is assumed to undergo steady state creep following Sherbys Law as given by,

$$
\epsilon=A\left(\left(\sigma-\sigma_{0}\right) / E\right)^{n} \exp (-Q / R T)
$$

The above equation can be written as,

$$
\epsilon=\left(M\left(\sigma_{e}-\sigma_{0}\right) / E\right)^{n},
$$

where, $M$ and $\sigma_{0}$ is the threshold stress and are known as creep parameters. The parameters $M$ and $\sigma_{0}$ depend on the reinforcement size, $P$, and the temperature of application, $T$. The values of $M$ and $\sigma_{0}$ in terms of material variables $P$ and operating temperature, $T$, have been extracted by using Sherbys law to describe the creep results obtained by Pandey et al [25].

The constitutive equations for creep in a rotating cylinder under multiaxial state of stress as given by Bhatnagar et al [15] and may be written as:

$$
\begin{aligned}
\epsilon_{r} & =\frac{\epsilon}{2 \sigma_{e}}\left((G+H) \sigma_{r}-F \sigma_{\theta}-H \sigma_{z}\right), \\
\epsilon_{\theta} & =\frac{\epsilon}{2 \sigma_{e}}\left((G+F) \sigma_{\theta}-F \sigma_{z}-H \sigma_{r}\right), \\
\epsilon_{z} & =\frac{\epsilon}{2 \sigma_{e}}\left((F+G) \sigma_{z}-G \sigma_{r}-F \sigma_{\theta}\right),
\end{aligned}
$$

\subsection{High temperature resin}

Here, we have discussed regarding the use of the high-temperature resins near the hot zones. Due to electrical heating near the bottom end cap, and aerodynamic heating near the stationary inserts, high-temperature resins needs to be used to avoid softening and delamination. Bismaleimides (BMIs), cyanate esters (CEs), benzoxazines and phthalonitriles group of resins are generally used to meet thermal challenges.

BMIs offer temperature performance and, because they cure by addition reaction rather than condensation reaction, they mitigate problems with emitted volatiles. For these reasons, BMIs have served on military aircraft for decades and into 5th Generation fighter planes, such as Lockheed Martins F-22 Raptor and the F-35 Lightning II Joint Strike Fighter. The Raptors airframe has served as an important test bed for BMI processing technology, and is about 25 percent composite by weight, split 50/50 between reinforced epoxy and BMI resins. The F-22s BMI accommodated application of resin transfer molding (RTM), including RTM internal ribs and spars, parts with $T$ of $I$ cross-sections. (The exterior skins are either hand layed or automated tape layed, using IM7/5250-4 BMI prepreg from Cytec Engineered Materials of Tempe, Ariz.) The closed-mold process produced parts with multiple finished surfaces that enabled secondary bonding, and its high injection pressure helped avoid ply bridging in deep draws and sharp corners. 


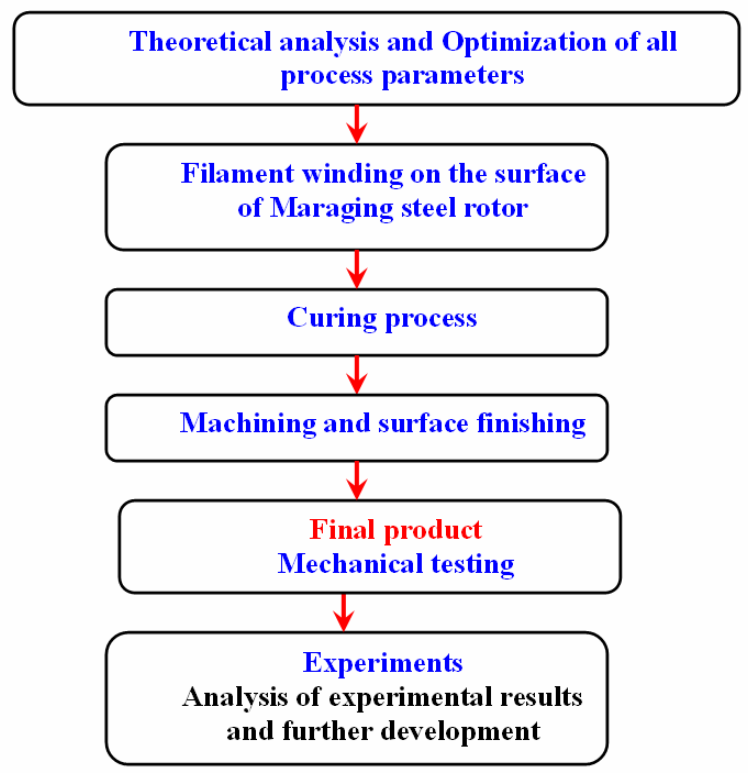

FiguRE 1. Major process steps for the filament winding of composite reinforced metallic cylinder. 


\section{REFERENCES}

Aksoy, S. \& Kurun, A. \& Cetin, E. \& Haboglu, M. R. 2014 Stress Analysis of Laminated Cylinders Subject to the Thermomechanical Loads. International Journal of Mechanical, Aerospace, Industrial and Mechatronics Engineering. 8, 242.

Bhavya, S. \& Kumar, P. R. \& Kalam, A. 2012 Failure Analysis of a Composite Cylinder. IOSR Journal of Mechanical and Civil Engineering (IOSR-JMCE). 3, 1.

Cohen, K. 1951 The Theory of Isotope Separation as Applied to the Large-Scale Production of $\mathrm{U}^{235}$.McGraw - Hill, New York, $195 \%$.

Gray, D. L. \& Moser, D. J. 2004 Finite Element Analysis of a Composit overwrapped pressure vessel. 40th AIAA/ASME/SAE/ASEE Joint Propulsion Conference and Exhibit Florida.

James \& WAng, T. S. \& Lin, C. C. 1993 Stresses in rotating composite cylindrical shells. Composite Structures. 25, 157.

Madhavi, M. \& RaO, K. V. J. \& RaO, K. N. 2009 Design and Analysis of Filament Wound Composite Pressure Vessel with Integrated-end Domes. Defence Science Journal. 59, 73.

Onder, A. 2007 First Failure Pressure Of Composite Pressure Vessels. Graduate School of Natural and Applied Sciences of Dokuz Eyll University.

Pecknold, D. A. \& Rahman, S. 1994 Micromechanics-based structural analysis of thick laminated composites. Computers and Structures. 51, 163.

Volety, I. V. 2006 Modeling Of Fiber Reinforced Polymer Confined Concrete Cylinders. Louisiana State University. 